\title{
Efficacy of CPAP modalities in lowering blood pressure in OSA: does the method used to measure blood pressure matter?
}

\author{
Babak Mokhlesi, ${ }^{1,2}$ Jason R Carter ${ }^{2,3}$
}

In recent years, a number of meta-analyses have consistently concluded that CPAP therapy elicits modest, yet significant, reductions in 24-hour ambulatory blood pressure (BP). ${ }^{1-4}$ These improvements in 24-hour BP appear to be more dramatic in patients with resistant hypertension, ${ }^{5}$ and reductions in nocturnal $\mathrm{BP}$ tend to be more dramatic than daytime BP. ${ }^{1-4}$ There is also evidence indicating that CPAP adherence plays a critical role in the potential BP-lowering effects of CPAP therapy. ${ }^{6}$

Pepin et $\mathrm{al}^{7}$ conducted the largest double-blind randomised-controlled trial (RCT) to date comparing the efficacy of 4 months of therapy with fixed-pressure CPAP (FP-CPAP, $\mathrm{n}=161$ ) versus autoadjusting CPAP (auto-CPAP, $n=161$ ) in reducing $\mathrm{BP}$ in patients with moderate-to-severe obstructive sleep apnoea (OSA). In an intention-to-treat analysis, there was no difference in the primary outcome of change in office systolic BP between FP-CPAP and auto-CPAP groups.

Auto-CPAP devices analyse flow and pressure signals on a breath-by-breath basis and use a variety of algorithms to adjust the delivered pressure in order to resolve detected residual obstructive events while trying to apply the lowest effective pressure. Thus far, the preponderance of data suggests that this modality is no better than FP-CPAP in improving adherence to therapy or quality of life. What remains unclear is whether the inherent fluctuations in delivered pressure by an auto-CPAP device can lead to microarousals and sleep fragmentation,

\footnotetext{
${ }^{1}$ Department of Medicine, Section of Pulmonary and Critical Care, Sleep Disorders Center, The University of Chicago, Chicago, Illinois, USA; ${ }^{2}$ Sleep, Metabolism and Health Center (SMAHC), The University of Chicago, Chicago, Illinois, USA; ${ }^{3}$ Department of Kinesiology and Integrative Physiology, Michigan Technological University, Houghton, Michigan, USA
}

Correspondence to Dr Babak Mokhlesi, Department of Medicine, Section of Pulmonary and Critical Care, Sleep Disorders Center, The University of Chicago, 5841 S. Maryland Ave, MC6076/Room M630, Chicago, IL 60637, USA; bmokhles@medicine.bsd.uchicago.edu ultimately leading to less effective reduction in nocturnal and daytime BP compared with FP-CPAP. Accordingly, the study by Pepin et al is important and timely, and the authors are to be commended for executing such a large and well-designed RCT to provide clinically useful information for the care of patients with OSA.

Pepin et $_{\mathrm{al}} \mathrm{l}^{7}$ estimated their sample size and set their a priori primary end point of office BP based on a prior smaller RCT by Patruno et $a l^{8}{ }^{8}$ which found a significant change in office BP with FP-CPAP but not with auto-CPAP after 3 months of therapy. It is important to note that in the study by Patruno et $a l^{8}$ the patients started with a significantly higher level of BP than the study by Pepin et al (mean BP $143 / 87$ vs $132.5 / 80 \mathrm{~mm} \mathrm{Hg}$, respectively). Moreover, $65 \%$ of the participants in the study by Pepin $e t a l^{7}$ were hypertensive at baseline. Although the number of subjects with hypertension was equally distributed between FP-CPAP and auto-CPAP groups, it is not clear whether equal numbers of patients in each group were on antihypertensive medications. Additionally, the timing of the antihypertensive medications is not specified. As this group of investigators has previously reported, the impact of antihypertensives on $\mathrm{BP}$ is much more robust than CPAP in patients with OSA, even on nocturnal BP. ${ }^{9}$ Therefore, the proportion of patients on antihypertensive medications, the timing of antihypertensives and the fact that the baseline office BP was relatively wellcontrolled could have potentially impacted the results, leading to no significant change in the primary outcome of office BP at 4 months. Alternatively, the effect size derived from Patruno et $a l^{8}$ may have been an overestimation, leading to underpowering of the study.

Despite the negative primary outcome, we believe that the secondary end points such as the 24-hour ambulatory BP findings and changes in BP based on perprotocol analysis have much more clinical relevance. Specifically, there was a greater reduction in diastolic BP with FP-CPAP in the intention-to-treat analysis. Moreover, in the per-protocol analysis, there was an even greater reduction in diastolic BP as well as in the mean BP during wakefulness. It is unclear why 24-hour diastolic BP was the only secondary outcome to reach significance, particularly given that the meta-analyses suggest that CPAP therapy tends to have its most robust influence on nocturnal systolic BP. This was not the case with the data from Pepin et $a l^{7}$ in fact, reductions in daytime BP seem to be the more likely driver for the greater 24-hour BP reductions with FP-CPAP than nocturnal BP. Regardless, the drop in diastolic BP may have important clinical implications since diastolic BP is also an important predictor of cardiovascular risk in middle-aged adults. ${ }^{10}$ The reason we believe per-protocol analysis is more relevant than the intention-to-treat analysis is due to the fact that 25 participants in the FP-CPAP and 16 participants in the auto-CPAP group withdrew from the study and did not have follow-up BP data at 4 months. Although we recognise that the investigators used appropriate and validated multiple imputation technique to estimate missing $\mathrm{BP}$ values to overcome this limitation, we believe that per-protocol analysis is more relevant.

In regards to adherence, the authors confirmed the finding from prior studies reporting similar levels of adherence between the two CPAP modalities. ${ }^{11}$ In the study by Pepin et $a l^{7}$ approximately $50 \%$ of patients used CPAP $\leq 5$ hours/ night. Although in clinical practice 4 hours of nightly CPAP use for $70 \%$ of the nights is considered adequate adherence to therapy, this translates into an average CPAP use of 2.8 hours every night. ${ }^{7}$ This is relevant in the context of rapid eye movement (REM) sleep, the stage of sleep associated with greater sympathetic activity and cardiovascular instability that prevails during the latter hours of normal nocturnal sleep. ${ }^{12-14}$ Analyses of two population-based studies have demonstrated that OSA during REM sleep is independently associated with prevalent and incident hypertension as well as with incident non-dipping of nocturnal BP. Notably these studies reported no significant association between obstructive apnoeas and hypopnoeas in non-REM sleep and hypertension. ${ }^{15-17}$ It is plausible that suboptimal CPAP adherence and the predominantly untreated OSA during REM sleep may explain the negative or modest effects of CPAP therapy on BP control in numerous RCTs. Indeed, using CPAP for 4 hours from the time lights are turned off will cover 
approximately $40 \%$ of REM sleep and will leave most obstructive events during REM sleep untreated. ${ }^{18}$

In contrast to the large number of studies examining the impact of CPAP therapy on office BP or on 24-hour ambulatory BP monitoring, there remains a paucity of studies examining the effect of CPAP therapy on nocturnal beat-to-beat BP. Although studies using 24-hour ambulatory BP monitoring have provided useful insight into the relationship between OSA and hypertension, this monitoring technique has inherent limitations because it typically measures BP in snapshots of 15-30 min intervals. Obstructive apnoeas and hypopnoeas can cause rapid and significant elevations of BP (ie, $\geq 30$ $40 \mathrm{mmHg}$ lasting 20-30 s) that might not be captured using ambulatory BP monitoring. Continuous beat-to-beat BP monitoring via finger plethysmography, on the other hand, offers a reliable non-invasive approach that can capture rapid oscillations and surges in BP that often occur during obstructive events. ${ }^{12}$ The few studies that have used beat-to-beat finger plethysmography before and after CPAP therapy provide unique mechanistic and clinical insights. For example, Bonsignore et $a l^{19}$ reported that 3-14 months of CPAP therapy improved nocturnal baroreflex function in patients with severe OSA. In a subsequent study, the same group demonstrated that as little as one night of CPAP significantly improved nocturnal baroreflex function. ${ }^{20}$ More recently, our laboratory used finger plethysmography to quantify the number of nocturnal surges in beat-to-beat BP in 17 patients with hypercapnic OSA. ${ }^{21}$ Indeed, in these patients who at baseline had relatively well-controlled BP during the daytime, there was no change in daytime office BP after 6 weeks of PAP therapy. However, there was a significant reduction in nocturnal BP as well as in the number of BP surges during sleep with PAP therapy. On average, these patients experienced nearly 50 systolic BP surges of $>40 \mathrm{~mm} \mathrm{Hg}$ per hour at night, but one night of PAP therapy reduced the number of surges by approximately $50 \%$, and 6 weeks of PAP therapy reduced the number of surges of $>40 \mathrm{~mm} \mathrm{Hg}$ by nearly $75 \% .^{21}$

In conclusion, the study by Pepin et $a l^{7}$ provides another piece of the puzzle in understanding the role of CPAP therapy on BP control in patients with OSA. While the office BP results did not demonstrate a difference between FP-CPAP and auto-CPAP, the authors observed a significantly greater reduction of BP in the FP-CPAP group when precision was enhanced through more frequent BP sampling using ambulatory BP monitoring. Perhaps additional insights could be gained from even more precision via finger plethysmography. Undoubtedly further research is needed to establish the clinical relevance of improving dramatic surges of BP during sleep with CPAP therapy in patients with OSA even if daytime BP does not change significantly. While we await the results of future trials using more robust measures of BP, Pepin et $\mathrm{al}^{7}$ have provided clear evidence that there is no significant difference between FP-CPAP and auto-CPAP on an important and practical clinical outcome such as office BP.

Competing interests $\mathrm{BM}$ is supported by National Institutes of Health grant R01HL119161 and has served as a consultant to Philips/Respironics and has received research support from Philips/Respironics. He has also received honorarium from Zephyr Medical Technologies and has served on the advisory board of Itamar Medical. JRC is supported by National Heart, Lung, and Blood Institute (HL-122919-01). BM and JRC are both supported by the Merck Investigator Studies Program.

Provenance and peer review Commissioned; externally peer reviewed.

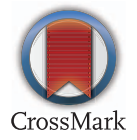

To cite Mokhlesi B, Carter JR. Thorax 2016;71:677678.

Published Online First 14 June 2016

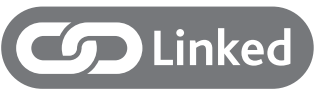

http://dx.doi.org/10.1136/thoraxjnl-2015-207700

Thorax 2016;71:677-678.

doi:10.1136/thoraxjnl-2016-208718

\section{REFERENCES}

1 Fava C, Dorigoni S, Dalle Vedove F, et al. Effect of CPAP on blood pressure in patients with OSA/ hypopnea a systematic review and meta-analysis. Chest 2014;145:762-71.

$2 \mathrm{Hu} \mathrm{X}$, Fan J, Chen S, et al. The role of continuous positive airway pressure in blood pressure control for patients with obstructive sleep apnea and hypertension: a meta-analysis of randomized controlled trials. J Clin Hypertens (Greenwich) 2015;17:215-22.

3 Montesi SB, Edwards BA, Malhotra A, et al. The effect of continuous positive airway pressure treatment on blood pressure: a systematic review and meta-analysis of randomized controlled trials. J Clin Sleep Med 2012;8:587-96.

4 Schein AS, Kerkhoff AC, Coronel CC, et al. Continuous positive airway pressure reduces blood pressure in patients with obstructive sleep apnea; a systematic review and meta-analysis with 1000 patients. J Hypertens 2014;32:1762-73.

5 Martínez-García MA, Capote F, Campos-Rodríguez F, et al., Spanish Sleep Network. Effect of CPAP on blood pressure in patients with obstructive sleep apnea and resistant hypertension: the HIPARCO randomized clinical trial. JAMA 2013:310:2407-15.

6 Barbé F, Durán-Cantolla J, Sánchez-de-la-Torre M, et al., Spanish Sleep and Breathing Network. Effect of continuous positive airway pressure on the incidence of hypertension and cardiovascular events in nonsleepy patients with obstructive sleep apnea: a randomized controlled trial. JAMA 2012;307: 2161-8.

7 Pepin J, Tamisier R, Baguet J, et al. Fixed pressure versus auto-adjusting CPAP: comparison of efficacy on blood pressure in obstructive sleep apnea, a randomized clinical trial. Thorax 2016;71:726-33.

8 Patruno V, Aiolfi S, Costantino G, et al. Fixed and autoadjusting continuous positive airway pressure treatments are not similar in reducing cardiovascular risk factors in patients with obstructive sleep apnea. Chest 2007;131:1393-9.

9 Pépin JL, Tamisier R, Barone-Rochette G, et al. Comparison of continuous positive airway pressure and valsartan in hypertensive patients with sleep apnea. Am J Respir Crit Care Med 2010;182:954-60.

10 Franklin SS, Larson MG, Khan SA, et al. Does the relation of blood pressure to coronary heart disease risk change with aging? The Framingham Heart Study. Circulation 2001;103:1245-9.

11 Ip S, D'Ambrosio C, Patel K, et al. Auto-titrating versus fixed continuous positive airway pressure for the treatment of obstructive sleep apnea: a systematic review with meta-analyses. Syst Rev 2012;1:20.

12 Somers VK, Dyken ME, Clary MP, et al. Sympathetic neural mechanisms in obstructive sleep apnea. J Clin Invest 1995;96:1897-904.

13 Somers VK, Dyken ME, Mark AL, et al. Sympathetic-nerve activity during sleep in normal subjects. N Engl J Med 1993;328:303-7.

14 Trinder J, Kleiman J, Carrington M, et al. Autonomic activity during human sleep as a function of time and sleep stage. J Sleep Res 2001;10:253-64.

15 Mokhlesi B, Finn LA, Hagen EW, et al. Obstructive sleep apnea during REM sleep and hypertension. results of the Wisconsin Sleep Cohort. Am J Respir Crit Care Med 2014;190:1158-67.

16 Mokhlesi B, Hagen EW, Finn LA, et al. Obstructive sleep apnoea during REM sleep and incident non-dipping of nocturnal blood pressure: a longitudinal analysis of the Wisconsin Sleep Cohort. Thorax 2015;70:1062-9.

17 Appleton SL, Vakulin A, Martin SA, et al. Hypertension is associated with undiagnosed obstructive sleep apnea during rapid eye movement (REM) sleep. Chest. Published Online First: 18 Mar 2016. doi:10.1016/j.chest.2016.03.010.

18 Grimaldi D, Beccuti G, Touma C, et al. Association of obstructive sleep apnea in rapid eye movement sleep with reduced glycemic control in type 2 diabetes: therapeutic implications. Diabetes Care 2014;37:355-63.

19 Bonsignore MR, Parati G, Insalaco G, et al. Continuous positive airway pressure treatment improves baroreflex control of heart rate during sleep in severe obstructive sleep apnea syndrome. Am J Respir Crit Care Med 2002;166:279-86.

20 Bonsignore MR, Parati G, Insalaco G, et al. Baroreflex control of heart rate during sleep in severe obstructive sleep apnoea: effects of acute CPAP. Eur Respir J 2006;27:128-35.

21 Carter JR, Fonkoue IT, Grimaldi D, et al. Positive airway pressure improves nocturnal beat-to-beat blood pressure surges in obesity hypoventilation syndrome with obstructive sleep apnea. Am J Physiol Regul Integr Comp Physiol 2016;310: R602-11. 